\title{
Current Closure in the Auroral Ionosphere: Results from the Auroral Current and Electrodynamics Structure Rocket Mission
}

S. R. Kaeppler, ${ }^{1}$ C. A. Kletzing, ${ }^{1}$ S. R. Bounds, ${ }^{1}$ J. W. Gjerloev, ${ }^{2}$ B. J.

Anderson, ${ }^{3}$ H. Korth, ${ }^{3}$ J. W. LaBelle, ${ }^{4}$ M. P. Dombrowski, ${ }^{4}$ M. Lessard ${ }^{5}$ R.

F. Pfaff, ${ }^{6}$ D. E. Rowland, ${ }^{6}$ S. Jones, ${ }^{6}$ C. J. Heinselman, ${ }^{7}$

${ }^{1}$ Department of Physics and Astronomy,

University of Iowa, Iowa City, Iowa, USA.

${ }^{2}$ Department of Physics and Technology,

University of Bergen, Bergen, Norway

${ }^{3}$ Applied Physics Laboratory, John

Hopkins University, Baltimore, Maryland,

USA.

${ }^{4}$ Department of Physics and Astronomy,

Dartmouth College, Hanover, New

Hampshire, USA.

${ }^{5}$ Space Sciences Center, University of New

Hampshire, Dover, New Hampshire, USA.

${ }^{6}$ NASA Goddard Space Flight Center,

Greenbelt, Maryland, USA. 
Abstract. The Auroral Current and Electrodynamics Structure (ACES) mission consisted of two sounding rockets launched nearly simultaneously from Poker Flat Research Range, AK on January 29, 2009 into a dynamic multiple-arc aurora. The ACES rocket mission was designed to observe electrodynamic and plasma parameters above and within the current closure region of the auroral ionosphere. Two well instrumented payloads were flown along very similar magnetic field footprints, at different altitudes, with small temporal separation between both payloads. The higher altitude payload (apogee $360 \mathrm{~km}$ ), obtained in-situ measurements of electrodynamic and plasma parameters above the current closure region to determine the input signature. The low altitude payload (apogee $130 \mathrm{~km}$ ), made similar observations within the current closure region. Results are presented comparing observations of the electric fields, magnetic components, and the differential electron energy flux at magnetic footpoints common to both payloads. In situ data is compared to the ground based all-sky imager data, which presents the evolution of the auroral event as the payloads traversed through magnetically similar regions. Current measurements derived from the magnetometers on the high altitude payload observed upward and downward field-aligned currents. The effect of collisions with the neutral atmosphere is investigated to determine

\footnotetext{
${ }^{7}$ Center for Geospace Studies, SRI

International, Menlo Park, California, USA.
} 
if it is a significant mechanism to explain discrepancies in the low energy electron flux. The high altitude payload also observed time-dispersed arrivals in the electron flux and perturbations in the electric and magnetic field components, which are indicative of Alfvèn waves. 


\section{Introduction}

Field-aligned currents and particle precipitation that cause the aurora, are two of the key mechanisms of the coupling that exists between the distant magnetosphere and the ionosphere. Upward directed field-aligned current sheets are associated with precipitating auroral electrons [Arnoldy, 1974; Elphic et al., 1998] that form inverted-V signatures in electron energy flux [Frank and Ackerson, 1971; Ackerson and Frank, 1972]. Black aurora [Marklund et al., 1994], a lack of visible auroral emission due to upgoing electrons, are typically associated with downgoing field-aligned currents which flow toward the ionosphere [Marklund et al., 1997; Elphic et al., 1998]. Within the lower ionosphere, currents flow perpendicular to the mean magnetic field that close the magnetospheric-ionospheric (MI) circuit by connecting the upward and downward field-aligned currents. Energy is transmitted from the magnetosphere to the ionosphere and is dissipated through Joule heating $\left(\mathbf{J}_{\perp} \cdot \mathbf{E}_{\perp}\right)$, where $\mathbf{J}_{\perp}$ is the perpendicular closure current. Energy may also be dissipated in the ionosphere by friction between with atmospheric neutrals and ions, which can be enhanced by auroral particle preciciption.

The perpendicular closure currents reside at altitudes where ion and electron collisions with the neutral atmosphere become significant. Therefore, these particles depart from their $\mathbf{E} \times \mathbf{B}$ drift motion, which invalidates the frozen-in approximation. The perpendicular current is established as a result of the cross-field velocity difference between ion and electron drifts. A useful parameter for determining the demagnetization of ions or electrons is $\kappa=\Omega_{\mathrm{s}} \nu_{\mathrm{s}}^{-1}$, which is the ratio of the cyclotron frequency $\left(\Omega_{\mathrm{s}}\right)$ to the collision frequency $\left(\nu_{\mathrm{s}}\right)$ [Sangalli et al., 2009]. When $\kappa$ is of the order of unity, then particle colli- 
sions begin to dominate thereby setting up the cross-field drift motion responsible for the Hall and Pederson currents. Ions begin to demagnetize at altitudes of less than $150 \mathrm{~km}$, yet electrons can remain tied to their cyclotron orbits to as low as $70 \mathrm{~km}$ [Richmond and Thayer, 2000].

Currents that flow perpendicular to the mean magnetic field, along channels of enhanced conductivity, are oriented in two directions relative to the perpendicular electric field, $\mathbf{E}_{\perp}$ : Pederson currents $\left(\mathbf{j}_{P}\right)$ flow parallel to $\mathbf{E}_{\perp}$, and Hall currents $\left(\mathbf{j}_{H}\right)$ flow perpendicular to $\mathbf{E}_{\perp}$. Ohm's law empirically relates the perpendicular electric field to the perpendicular current density, as represented by Richmond and Thayer [2000]:

$$
\mathbf{j}_{\perp}(z)=\mathbf{j}_{P}(z)+\mathbf{j}_{H}(z)=\sigma_{P}(z) \mathbf{E}_{\perp}+\sigma_{H}(z)\left(\hat{\mathbf{B}} \times \mathbf{E}_{\perp}\right)
$$

where $\hat{\mathbf{B}}$ is the magnetic field unit vector. The height dependent Hall and Pederson conductivities [Cowling, 1945], $\sigma_{\mathrm{H}}(\mathrm{z})$ and $\sigma_{\mathrm{P}}(\mathrm{z})$, are described by Richmond and Thayer [2000] as equations (2) and (3), respectively:

$$
\begin{aligned}
& \sigma_{H}(z)=\frac{n_{e} e}{B}\left(\frac{\Omega_{e}^{2}}{\nu_{e n}^{2}+\Omega_{e}^{2}}-\frac{\Omega_{i}^{2}}{\nu_{i n}^{2}+\Omega_{i}^{2}}\right) \\
& \sigma_{P}(z)=\frac{n_{e} e}{B}\left(\frac{\nu_{e n} \Omega_{e}}{\nu_{e n}^{2}+\Omega_{e}^{2}}+\frac{\nu_{i n} \Omega_{i}}{\nu_{i n}^{2}+\Omega_{i}^{2}}\right)
\end{aligned}
$$

where $\nu_{\mathrm{sn}}$ is the collision frequency between species (s) with atmospheric neutrals. The Pederson conductivity peaks at higher altitudes of approximately $130-150 \mathrm{~km}$, and the Hall conductivity peaks at altitudes below $120 \mathrm{~km}$ [Richmond and Thayer, 2000].

Assuming space charge does not accumulate, the current continuity equation can be solved to relates field-aligned current $\left(\mathrm{j}_{\|}\right)$to perpendicular currents $\left(\mathbf{j}_{\perp}\right)$. A commonly invoked assumption is to assume that the Hall and Pederson conductivities are constant 
over some altitude range [Fejer, 1953; Swift, 1972] and can be integrated to yield:

$$
j_{\|}=\int\left(-\nabla \cdot \mathbf{j}_{\perp}(z)\right) d z=\Sigma_{P} \nabla_{\perp} \cdot \mathbf{E}_{\perp}+\Sigma_{H} \nabla_{\perp} \cdot\left(\hat{\mathbf{B}} \times \mathbf{E}_{\perp}\right)
$$

where $\Sigma_{\mathrm{H}}$ and $\Sigma_{\mathrm{P}}$ are the height integrated Hall and Pederson conductivities, respectively. Equation (4) forms the fundamental equation that relate field-aligned current to the perpendicular closure current in the ionosphere.

Auroral particle precipitation also affects the Hall and Pederson conductivities in the current closure region. Deposition of precipitating electrons that form the aurora and ionize atmospheric neutrals can substantially increase the local electron plasma density, which increases the Hall and Pederson conductivities. Rees [1963] derived ionization models that were functions of altitude and included different incident electron flux distributions onto the ionosphere, valid for electrons with energies of 0.4-300 KeV. Rees [1982] later derived similar ionization models for protons. Boström [1964] was one of the first to include the altitude dependence of the electron density, consistent with the results of Rees [1963], in a derivation of the Hall and Pederson conductivities. A significant increase in Hall and Pederson conductivities was found at approximately $100 \mathrm{~km}$; this result suggested that precipitating electrons augment the development of an altitude layer that enhance the flow of cross field currents.

The low altitude of the aurora and the ionosphere make it particularly well-suited for observations by sounding rockets. Many sounding rocket missions have examined electrodynamics associated with the aurora [Arnoldy, 1974, 1977; Evans et al., 1977; Marklund et al., 1982; Mallinckrodt and Carlson, 1985; Kletzing et al., 1996; Sangalli et al., 2009]. Sounding rocket data analyzed by Evans et al. [1977] presented one of the most complete studies of auroral electrodynamics. Evans et al. [1977] calculated Hall and Pederson con- 
ductivities from observed electron densities, and found both conductivities significantly increased as the payload traversed a stable auroral arc. Evans et al. [1977] observed a strong correlation between a reduced perpendicular electric field magnitude and a similar reduction in the magnitude of the reciprocal of the height integrated Pederson conductivity over the auroral arc. This observation suggested that increased Hall and Pederson conductivities will result in reduced electric field magnitude within the auroral arc. Further sounding rocket missions [Mallinckrodt and Carlson, 1985; Marklund et al., 1982; Kletzing et al., 1996] found similar anticorrelations between the electric field magnitude and the height integrated Hall and Pederson conductivity that were consistent with the observations of Evans et al. [1977].

A model was developed by Mallinckrodt [1985] that examined the altitudinal and latitudinal current density structure within the auroral ionosphere $(80-250 \mathrm{~km})$ to assist in explaining anticorrelation observations made by Mallinckrodt and Carlson [1985]. Input electric fields and field-aligned currents were prescribed at the upper boundary of the model. Two different auroral electrodynamic configurations, as classified by Marklund [1984], were used: polarization arcs, in which space charge separation creates a polarization electric field with negligible field-aligned currents, and field-aligned current arcs, in which significant field-aligned currents were present. The model included other significant effects such as ionospheric particle density, ionization profiles, and neutral winds. In the case of polarization arcs, the model suggested a vortex in current would develop in the auroral ionosphere; whereas, in the case of field-aligned current arcs the closure geometry was U-shaped. These results in Mallinckrodt [1985] suggested that the altitu- 
dinal and latitudinal current closure geometries depend strongly upon the input auroral configuration.

Energy from the magnetosphere is transmitted into the ionosphere via precipitating particles and through the Poynting flux $\mathbf{E} \times \delta \mathbf{B}(\delta \mathbf{B}$ is a perturbation with respect to the mean magnetic field). If a steady state configuration has been achieved, Poynting's theorem states that electromagnetic energy carried by the Poynting flux will be dissipated through Joule heating $\left(\mathbf{J}_{\perp} \cdot \mathbf{E}_{\perp}\right)$ [Cowley, 1991; Richmond and Thayer, 2000]. In the frame of the plasma, closure current, that is enhanced by precipitating particles, will also be dissipated through Joule heating. Joule heating is expressed in the following form:

$$
\mathbf{J}_{\perp} \cdot \mathbf{E}_{\perp}=\mathbf{J}_{P} \cdot \mathbf{E}_{\perp}=\Sigma_{P} \mathbf{E}_{\perp}^{2}
$$

where $\mathbf{J}_{P}$ is the Pederson current, $\Sigma_{\mathrm{P}}$ is the height integrated Pederson conductivity, and $\mathbf{E}_{\perp}$ is the perpendicular electric field in the plasma or neutral wind frame of reference. The Hall current does not contribute to the transfer of energy, even though the Hall current is typically more significant at altitudes below the Pederson current [Evans et al., 1977; Fujii et al., 1998; Sangalli et al., 2009]. Calculations of Joule heating are typically undertaken in the neutral wind frame of reference. The neutral wind can contribute as an additional dissipation mechanism if ions become tied to the neutral wind velocity, then friction between ions and neutrals will be significant [Richmond and Thayer, 2000; Fujii et al., 1998]. Vasyliūnas and Song [2005] have argued that Joule heating calculations in the neutral frame are a matter of convention and that Joule heating should be calculated in the plasma frame of reference, rather than the neutral wind frame.

Several sounding rocket missions have reported measurements of the Hall and Pederson conductivities that were used to calculate the Joule heating rate, particularly over auroral 
arcs [Evans et al., 1977; Marklund et al., 1982; Mallinckrodt and Carlson, 1985; Kletzing et al., 1996]. However, few observations have been reported of in situ Joule heating measurements within the current closure region of the ionosphere. The JOULE II sounding rocket mission examined the low altitude auroral ionosphere and neutral atmosphere, particularly, the interplay between the neutral wind and the demagnetization of ions [Sangalli et al., 2009]. Sangalli et al. [2009] also presented in situ ion flow velocities, the $\mathbf{E} \times \mathbf{B}$ drift velocities, the electron density, and direct calculations of the Hall and Pederson conductivities from these observed parameters. The JOULE II rocket mission also included additional chemical release payloads to measure neutral wind velocities valid for altitudes of 90 to $130 \mathrm{~km}$. Sangalli et al. [2009] found that the in situ Joule heating rate peaked at approximately $114 \mathrm{~km}$ and, including the neutral wind effects, the Joule heating rated decreased by $28 \%$. The reduction in the Joule heating rate including the neutral wind suggested that some of the energy was dissipated through ion-neutral frictional drag. The results from the JOULE II mission present single point measurements of various parameters within the current closure region of the ionosphere, but did not have simultaneous observations of the magnetospheric sources of particles and electromagnetic fields that map down to the ionosphere.

In order to understand plasma and electrodynamic observations within the current closure region, one has to examine how the magnetospheric sources interplay with the ionosphere. Observations of phenomena such as current closure geometry and Joule heating require additional observations of the magnetospheric inputs. In addition, within the current closure ionosphere, it is common to integrate out the height dependence of the Hall and Pederson conductivities. However, while the assumption that the Hall and Pederson 
conductivities are constant with respect to altitude is acceptable for large scale models, it does not hold for detailed models within the current closure region. Incoherent scatter radar observations made by Fujii et al. [1998] suggested that even over a small height range of $20 \mathrm{~km}$, there were significant differences in the height integrated conductivities. These observational issues motivated a twin-payload sounding rocket mission that would cross similar magnetic field footpoints, at differing altitudes, to obtain in situ observations in the current closure region while also observing the magnetospheric input signatures.

\section{Mission and Instrumentation}

The Auroral Currents and Electrodynamics Structure (ACES) rocket mission utilized two nearly identical, well-instrumented payloads that flew along similar magnetic field footpoints with small longitudinal separation to measure key electrodynamic fields and plasma parameters. Both payloads were launched nearly simultaneously to constrain the temporal-spatial ambiguity inherent of in situ observations. The first payload (hereafter referred to as "ACES High") was designed to fly at higher altitudes to measure electrodynamic and plasma inputs into the current closure region. The second lower altitude payload (hereafter referred to as "ACES Low") was designed to obtain in situ plasma and electrodynamic observations within the current closure region. In addition to the rocketbourne payloads, all-sky imagers and the Poker Flat Incoherent Scatter Radar (PFISR) provided photometric and radar observations of the auroral configuration, respectively. The combined ground based and in situ observations make the ACES mission one of the most comprehensive data sets to examine the altitudinal structure of an auroral arc and the current closure system. 
ACES High and Low successfully launched from Poker Flat Research Range, Alaska on January 29, 2009 at 09:49:40.0 UT and 09:51:10.0 UT, respectively. The overall geomagnetic conditions preceding the launch were very quiet as a result of the launch window being near solar minimum. The payloads were launched into a dynamic multiple-arc aurora located north of Ft. Yukon, the approximate apogee location of both payloads. Ground based magnetometers indicated a $100 \mathrm{nT}$ deflection in the H-component observed at Ft. Yukon. This deflection suggested that the event was a substorm with an electrojet, which indicates a current closure system was established in the arc. The launch time of both payloads was approximately 2300 MLT which was in the evening-midnight MLT time sector. The Altitude Adjusted Corrected Geomagnetic Model (AACGM) was used to produce the magnetic mapping presented in Figure 1 which shows when the payloads crossed similar magnetic field lines that map down to ionospheric footpoints at an altitude of $110 \mathrm{~km}$. ACES High reached an apogee of $360 \mathrm{~km}$, whereas ACES Low reached 130 $\mathrm{km}$. The maximum longitudinal separation, that mapped down to footpoints at $110 \mathrm{~km}$, between both payloads was $23 \mathrm{~km}$, near the end of their respective trajectories.

\subsection{Instrumentation}

The ACES payloads included a variety of instruments to measure auroral plasma electron populations. The Electrostatic Electron Pitch-Angle Analyzer (EEPAA) was used to obtain full pitch angle differential energy flux distributions of precipitating auroral electrons. The EEPAA is a "top-hat" style electrostatic particle analyzer [Carlson et al., 1982] consisting of 24 anode pads simultaneously measuring $15^{\circ}$ pitch angle bins, while the symmetry axis of the detector remains nominally aligned to the mean magnetic field. A full energy sweep of $0.1-16 \mathrm{KeV}$ was completed in $48 \mathrm{~ms}$ to create full electron distri- 
butions with high temporal resolution. An electron retarding potential analyzer (ERPA) was aligned parallel to the spin-axis of the payload to measure the differential electron energy flux of the background $(0-3 \mathrm{eV})$ electron populations and the electron temperature. For a payload that is well-aligned to the mean magnetic field, the ERPA can make observations of the low energy field-aligned electrons that carry the current. Spherical swept and fixed biased Langmuir probes [Schott, 1968] suspended on a boom $1 \mathrm{~m}$ from the payload and an impedance probe [Jensen and Baker, 1992] were used to obtain in situ electron density measurements. Data from the Langmuir probe, impedance probe, and PFISR radar provide the capability for cross calibration between instruments to arrive at a precise absolute electron density.

The ACES payloads also included a suite of instruments to measure electric and magnetic fields within the plasma. A high resolution fluxgate magnetometer (Acuña [2002] and references therein) was aligned with the spin-axis of the payload to obtain DC magnetic field measurements. Due to payload spin and coning motion, the fluxgate magnetometer could observe magnetic perturbations, which are indicative of current sheets, resolved down to better than $50 \mathrm{nT}$ over the background 50,000 nT magnetic field. The double probe technique $[P f a f f, 1996]$ was used to obtain the low frequency and DC electric fields $(0-5 \mathrm{KHz})$ perpendicular to the spin-axis of the payload. The double probe spherical sensors were attached to booms with tip-to-tip length of $5.3 \mathrm{~m}$ and $3.0 \mathrm{~m}$ on ACES High and Low, respectively. AC double probes were aligned along the spin-axis of the payload to measure high frequency (HF) electric fields with a bandwidth of 0-5 MHz. Cutoffs in the plasma frequency $\mathrm{f}_{\mathrm{pe}}$ measured by the HF double probes can be used as an additional cross calibration of the electron density. 
The mission was successful, but a few issues arose on each payload during flight. Both payloads were spinning and the spin-axis had a coning precession relative to the mean magnetic field. The ACES High payload coned with a half angle of $3^{\circ}$, but ACES Low coned at a much larger half angle of $13^{\circ}$. This larger coning precession was due to a valve leak in the attitude control system gas tank. The EEPAA on ACES High had a failure in the energy sweep electronics board, that truncated the full sweep range. Therefore, the peak energy observed on ACES High was only $500 \mathrm{eV}$, as opposed to the full $16 \mathrm{KeV}$.

\section{Results}

Figure 2 presents the relevant electrodynamic and electron plasma data for both ACES High and Low, respectively. The data was obtained from the EEPAA, DC double probes, and the fluxgate magnetometer to examine the electrodynamics of the auroral configuration. The top panel of Figure 2 is a spectrogram of the differential electron energy flux, as a function of energy in $\mathrm{eV}$ and time in UT. The two middle panels in Figure 2 are the zonal (east-west) and meridional (north-south) DC electric fields in units of $\mathrm{mVm}^{-1}$ after being transformed into the geophysical East-North-Up (ENU) coordinate system. The final three panels in Figure 2 present the ENU residual DC magnetic field components in $\mathrm{nT}$ after a third-order polynomial fit was applied to remove the mean magnetic field. For the ACES Low data presented in Figure 2, the position of the ACES Low payload was magnetically mapped down to the $110 \mathrm{~km}$ footpoints that were previously crossed by ACES High during the time interval 09:54:20 UT - 09:55:20 UT. Figure 3 presents a montage of six all-sky images, from the Fort Yukon imager, depicting the auroral evolution. These images show the magnetic footpoints of the ACES High and Low payloads, especially during the regions of conjugate data coverage between both payloads. 


\subsection{Electron Flux}

The spectrograms of the differential electron energy flux shown in the top panel of Figure 2 for both ACES High and Low cover the pitch angle range of $15-30^{\circ}$. This pitch angle range selected because it was nearly field-aligned, while remaining relatively immune to data losses caused by payload coning motion. The gray bands in the ACES Low differential electron energy spectrum in Figure 2 are regions in which data was unavailable.

Although the full energy range was not observed, the differential electron energy flux is ideal for an analysis focused on low energy precipitating auroral electrons. The data from the all-sky imager have been compared against the differential electron energy flux to gain insight into the auroral event ACES High traversed. ACES High had three auroral crossings over the course of the flight. As shown in Figure 3A, the payload entered a relatively stable auroral arc at 09:53:20 UT and exited the visible region at approximately 09:54:00 UT. There was a modest increase in the differential electron energy flux at approximately 09:53:20 UT which correlates well with the entry of the payload into the first quasi-stable, visible arc. At 09:54:00 UT there was a depletion of low energy electron flux that correlates well with the time when the payload entered the dark region adjacent to the northward edge of the quasi-stable arc, as shown in Figure 3B.

At approximately 09:54:15 UT, ACES High passed over a faint auroral arc in the all-sky imager, as shown in Figure 3C. At this time, a time-dispersed arrival of low energy electrons was observed in the differential electron energy flux. These time-dispersed electron events are similar in nature to the results of Kletzing and $H u$ [2001] who showed through simulation that electrons accelerated by Alfvèn waves produced a time-dispersed signature, especially for electrons with energies less than $1 \mathrm{KeV}$. Although measurements of 
inverted-V electrons are not available, Chen et al. [2005] further showed through simulation that time-dispersed electron events can occur simultaneously with inverted- $\mathrm{V}$ energy precipitating electrons. The all-sky imager data in Figure 3D indicates that ACES High glanced the eastward edge of a large region of dynamic eastward-moving aurora. The differential electron energy flux data indicated that there were moderate, low energy precipitation in this region along with embedded regions of increased electron flux and isolated time-dispersed electron precipitation.

By 09:55:15 UT ACES High had exited the second auroral region and had moved into a region that was devoid of visible aurora and precipitating electrons, as shown in Figure 3E. However, isolated time-dispersed electron events, such as the event at 09:55:45 UT, were observed which do not correlate with visible aurora in the all-sky imagers and may be associated with sub-visual auroral arcs. At 09:56:15 UT the all-sky imager in Figure 3F showed that ACES High encountered the final auroral crossing, into a very active poleward arc. The differential electron energy flux data further indicated that after passing into a region of depleted flux, the payload entered into a region of intense time-dispersed electron precipitation.

The differential electron energy flux for ACES Low indicated that the payload traversed two inverted-Vs that were located on similar magnetic field flux tubes that were previously crossed by ACES High. As shown in Figure 3B, ACES Low began to cross the quasistable arc at 09:54:00, 40 seconds after ACES High had previously passed through the same region. Figure $3 \mathrm{C}$ shows that the visible arc remained spatially stable; however, it began to fade in intensity as the ACES Low payload completed its passage through the arc, exiting at 09:54:30 UT. The correlation between the the differential electron energy 
flux on ACES Low and the all-sky images suggest that the quasi-stable arc produced an inverted-V with a peak energy of $4 \mathrm{KeV}$. At 09:54:35 UT, ACES Low passed through a region of reduced visible emission in the all-sky imager, which correlated well with a region of reduced electron flux. At 09:54:40 UT ACES Low began to enter the eastern edge of the eastward-moving auroral form that ACES High had previously glanced, as illustrated by Figure 3D. ACES Low moved through a much large portion of the dynamic eastward-moving region, as illustrated by Figure 3E. An inverted-V was observed in the differential electron energy flux with a similar peak energy of $4 \mathrm{KeV}$ that corresponds well with eastward-moving region.

\subsection{Electric Fields}

The DC electric fields presented in Figure 2 have had the effects of payload coning and spin removed and are presented in the ENU geophysical coordinate system. These electric fields observed on ACES High and Low are generally southward and westward. For the midnight MLT time sector, into which the payloads were launched, the electric fields observed on ACES High and Low are consistent with electric fields formed as a result of plasma convection around the Earth [Baumjohann, 1982].

On ACES High, the zonal component of the electric field shows little variation until approximately 09:55:45 UT. Based on the all-sky images, the ENU coordinate system appears to be fairly well aligned with the arc frame. Therefore, the electric field observations in the zonal component are consistent with the continuity of electric fields across the arc boundary. The southward directed field exhibited more variability, but this component returned to a fairly constant southward direction until 09:55:45 UT. As the payload entered the region with significant time-dispersed electron events after 09:55:45 UT, both 
of the electric field components reversed direction to more eastward and northward. Significant electric field perturbations also correlated well with the time-dispersed arrival of electrons, after 09:55:45 UT and in earlier events, such as 09:54:15 UT. The correlation of these electric field perturbations with the time-dispersed electrons provide further evidence that Alfvèn waves were present.

The DC electric fields on the ACES Low payload also showed low levels of variation over the duration of the flight. The westward directed electric field exhibited a maximum variation of $15 \mathrm{mVm}^{-1}$; whereas, the southward directed field had a maximum variation of $20 \mathrm{mVm}^{-1}$. The oscillations at the beginning of the data, in both components of the electric field, were an artifact of the payload attitude solution. One mechanism that could explain observations of the electric fields observed on ACES Low is as follows: for a closure current that was relatively constant, an increase in conductivity due to the low altitude of the payload, would result in a decrease in the electric field magnitude [Evans et al., 1977; Kletzing et al., 1996].

\subsection{Magnetic Fields}

The bottom three panels in Figure 2 present the residual magnetic field for both ACES High and Low, after the mean Earth magnetic field has been subtracted. Both payloads observed variations in all residual magnetic field components of magnitude less than 150 nT . On ACES High before 09:56:00 UT, variations in the eastward and northward residual magnetic fields components were suggestive that field-current sheet currents were present. The event at 09:54:00 UT correlates well with EEPAA observations of depletions in differential electron energy flux that could result from an upward directed return current [Elphic et al., 1998]. One of the most notable signatures of the presence of field-aligned 
currents occurred at 09:56:00 UT, in which there was a $120 \mathrm{nT}$ reversal from north to south, while simultaneously, a reversal of $100 \mathrm{nT}$ from west to east was observed. This event at 09:56:00 UT was indicative of a large magnitude field-aligned current sheet. Furthermore, observations of depletions in the precipitating differential electron energy flux were observed at the location of this magnetic reversal. As a final signature regarding the presence of Alfvèn waves, perturbations on the order of $20 \mathrm{nT}$ were observed in the residual magnetic field components at the same time as similar perturbations in the electric field and the observations of time-dispersed low energy electrons in the differential electron energy flux.

The ACES Low magnetometer has retained small amplitude spin and coning components in the residual magnetic field magnitudes. The remaining coning and spin amplitudes are on the order of $15 \mathrm{nT}$ and on the order of a spin period in duration. However, larger-scale gradients in the residual magnetic fields that may be indicative of currents, particularly in the eastward component, correlate well with a depletion in the differential electron energy flux at approximately 09:54:30 UT. Figure 3D shows that ACES Low passed by a region that is devoid of visible aurora. It is plasuible that this magnetic field gradient, along with a lack of visible aurora and lack of differential electron energy flux could suggest that some component of the total current is a downward field aligned current. Shortly thereafter, at 09:54:50 UT, a $150 \mathrm{nT}$ reversal in the eastward directed component also correlates well with precipitating electrons associated with the large eastward-moving auroral region. At this point, the ACES Low may be moving into another region with some component of an upward field aligned current. 


\subsection{Currents}

A calculation of the field-aligned currents was performed based on the magnetic perturbations on ACES High. A sheet approximation was used to calculate field-aligned current components from the residual magnetic field perturbations. Assuming that the field-aligned current was aligned with the Up direction in the ENU frame, equation (6) was used to determine the field-aligned current from the magnetic field perturbations:

$$
j_{U}=\frac{1}{\mu_{0}}(\nabla \times \mathbf{B})_{U}=\frac{1}{\mu_{0}}\left(\frac{\Delta B_{N}}{v_{E} \Delta t}-\frac{\Delta B_{E}}{v_{N} \Delta t}\right)
$$

where $\mathrm{v}_{\mathrm{i}}$ indicates the track velocity of the payload and $\Delta \mathrm{t}$ was the time interval between successive samples. Figure 4 illustrates the result of this calculation, in which the differential electron energy flux is plotted above the field-aligned current for comparison. Observations on ACES High agree with results from Arnoldy [1977], in which it was found that currents tend to be observed toward the edges of auroral arc structures. In addition, upward field-aligned current regions, indicative of downward moving electrons were observed over regions mapped to inverted-V aurora as deduced from ACES Low data and the regions of visible aurora on the all-sky imagers. A lack of electron flux correlated well with regions of downward field-aligned current, which is consistent with upward moving electrons [Marklund et al., 1994, 1997; Elphic et al., 1998].

\section{Discussion}

The lack of structure in the low energy differential electron energy flux was one of the most notable differences between observations made on ACES High verse ACES Low. Two explanations can account for this discrepancy. First, the auroral configuration evolved during the time interval when ACES High first transversed a given region to the time 
when ACES Low passed through the same region. While the first auroral arc was quasistable over the time interval of both payload flights, the larger eastward-moving auroral region was dynamically evolving over short time scales.

The second explanation was that precipitating electrons colliding with atmospheric neutrals become significant at ACES Low altitudes. To determine the effect of ionospheric collisionality, the stopping altitude $\mathrm{h}_{\mathrm{s}}$ was determined through a procedure similar to that described in Kivelson and Russell [1995], section 7.2.2. The stopping altitude is determined by iteratively integrating equation (7):

$$
\int_{\eta\left(h_{s}\right)}^{\infty} \rho_{n}(h) d h=R\left(\xi_{0}\right)
$$

to find the height that corresponds to the value of $\eta\left(\mathrm{h}_{\mathrm{s}}\right)$, the altitude dependent mass density, that is equal to the range energy function:

$$
R\left(\xi_{0}\right)=4.3 \times 10^{-7}+5.36 \times 10^{-6} \xi_{0}^{-1.67}
$$

The range energy function $\left(\mathrm{R}\left(\xi_{0}\right)\right)$ is an experimentally determined function which relates an incident electron to the mean distance the particle will travel before scattering to the extent that it is indistinguishable from the background electron population. The range energy function, equation (8) was defined by Rees [1989] and a model ionospheric neutral mass density $\left(\rho_{\mathrm{n}}\right)$ was used [Kelley, 1989]. Figure 5 presents the incident energy of precipitating electrons verses altitude; it was found from this calculation that electrons at $500 \mathrm{eV}$ were typically scattered and became indistinguishable from the background plasma at approximately $160 \mathrm{~km}$. This is $30 \mathrm{~km}$ above the apogee of ACES Low, which suggests that collisions are significant enough to diminish structure in the precipitating low energy differential electron energy flux. 
One of the other notable differences between ACES High and Low resides in the magnitude of the residual Up magnetic field component. If it is assumed that the Up component is nominally aligned antiparallel to the direction of the mean magnetic field, then components that were field-aligned would only contribute to gradients in the eastward and northward residual magnetic field . The gradients in the Up component would be negligible. However, if cross field currents flow, then the Up component of the residual magnetic field would become significant. To gain a figure of merit as to the importance of the the Up component, the deviation between the maximum and minimum peaks in the Up component are compared between ACES High and Low. On ACES High the maximum magnetic variation observed between peaks in the Up component are of the order of $50 \mathrm{nT}$; however, on ACES Low the maximum variation observed between peaks is approximately $200 \mathrm{nT}$, a factor of four larger than on ACES High. This variation in the Up component suggests that ACES Low cross-field currents are significant. A more sophisticated geometry will be required to evaluate the currents observed by ACES Low and it is outside the scope of this paper.

\section{Conclusions}

Two payloads were successfully launched from Poker Flat Research Range, Alaska, on January 29, 2009 into a dynamic multiple-arc aurora. Both payloads crossed similar magnetic field lines mapped down to footpoints at $110 \mathrm{~km}$, with small longitudinal separation and observations between payloads were separated by less than a minute. There is a approximately a minute of conjugate data coverage between both payloads. Both pay-

loads traversed a quasi-stable arc early in their respective flights and later traversed an 
eastward-moving auroral form. Toward the end of the flight, ACES High crossed a very dynamic poleward arc.

Data presented for both payloads included the differential electron energy flux, electric fields and residual magnetic fields. Field-aligned currents were also derived from residual magnetic field components for the ACES High payload. ACES High observed time-dispersed arrivals of low energy precipitating electrons and simultaneous observations of perturbations in the electric and magnetic fields. These observations suggest that ACES High may have traversed regions where Alfvèn waves were present. Upward and downward field-aligned sheet currents were observed and compared to both the differential electron energy flux and all-sky images. It was found that regions with a lack of precipitating electrons and lack of visible aurora in the all-sky imagers were observed at the same time as regions of downward directed field-aligned current. Conversely, regions with precipitating electron flux correlated very well with upward field-aligned currents.

ACES Low traversed two inverted-Vs with peak energies of $4 \mathrm{KeV}$. One of the most significant features was the lack of structure in the low energy precipitating electrons. To get a measure for how significant collisional effects might be on ACES Low, a calculation of the stopping altitude suggested that most electrons below $1 \mathrm{KeV}$ would appear as indistinguishable from the ionospheric background. Ionospheric conductivity may be the dominant mechanism responsible for the low variation in the magnitude of both of the electric field components. Perturbations in the residual magnetic field components are strongly suggestive that currents were flowing at ACES Low altitudes. 


\section{References}

Ackerson, K. L., and L. A. Frank, Correlated satellite measurements of low-energy electron precipitation and ground-based observations of a visible auroral arc., J. Geophys. Res., 77, 1128-1136, doi:10.1029/JA077i007p01128, 1972.

Acuña, M. H., Space-based magnetometers, Review of Scientific Instruments, 73, 37173736, doi:10.1063/1.1510570, 2002.

Arnoldy, R. L., Auroral particle precipitation and Birkeland currents., Reviews of Geophysics and Space Physics, 12, 217-231, 1974.

Arnoldy, R. L., The relationship between field-aligned current, carried by suprathermal electrons, and the auroral arc, Geophys. Res. Lett., 4, 407-410, doi: 10.1029/GL004i010p00407, 1977.

Baumjohann, W., Ionospheric and field-aligned current systems in the auroral zone - A concise review, Advances in Space Research, 2, 55-62, doi:10.1016/0273-1177(82)903635,1982 .

Boström, R., A Model of the Auroral Electrojects, J. Geophys. Res., 69, 4983-4999, doi:10.1029/JZ069i023p04983, 1964.

Carlson, C., D. Curtis, G. Paschmann, and W. Michel, An instrument for rapidly measuring plasma distribution functions with high resolution, Advances in Space Research, 2(7), 67 - 70, doi:DOI: 10.1016/0273-1177(82)90151-X, 1982.

Chen, L.-J., C. A. Kletzing, S. Hu, and S. R. Bounds, Auroral electron dispersion below inverted-V energies: Resonant deceleration and acceleration by Alfvén waves, Journal of Geophysical Research (Space Physics), 110, A10S13, doi:10.1029/2005JA011168, 2005. 
Cowley, S. W. H., Acceleration and heating of space plasmas - Basic concepts, Annales Geophysicae, 9, 176-187, 1991.

Cowling, T. G., The Electrical Conductivity of an Ionized Gas in a Magnetic Field, with Applications to the Solar Atmosphere and the Ionosphere, Royal Society of London Proceedings Series A, 183, 453-479, doi:10.1098/rspa.1945.0013, 1945.

Elphic, R. C., et al., The auroral current circuit and field-aligned currents observed by FAST, Geophys. Res. Lett., 25, 2033-2036, doi:10.1029/98GL01158, 1998.

Evans, D. S., N. C. Maynard, J. Troim, T. Jacobsen, and A. Egeland, Auroral vector electric field and particle comparisons. II - Electrodynamics of an arc, J. Geophys. Res., 82, 2235-2249, doi:10.1029/JA082i016p02235, 1977.

Fejer, J. A., Semidiurnal currents and electron drifts in the ionosphere, Journal of Atmospheric and Terrestrial Physics, 4(4-5), 184 - 203, doi:10.1016/0021-9169(53)90054-3, 1953.

Frank, L. A., and K. L. Ackerson, Observations of charged particle precipitations into the auroral zone., J. Geophys. Res., 76, 3612-3643, doi:10.1029/JA076i016p03612, 1971.

Fujii, R., S. Nozawa, N. Matuura, and A. Brekke, Study on neutral wind contribution to the electrodynamics in the polar ionosphere using EISCAT CP-1 data, J. Geophys. Res., 1031, 14,731-14,740, doi:10.1029/97JA03687, 1998.

Jensen, M. D., and K. D. Baker, Measuring ionospheric electron density using the plasma frequency probe, Journal of Spacecraft and Rockets, 29, 91-95, doi:10.2514/3.26318, 1992.

Kelley, M. C., The earth's ionosphere : plasma physics and electrodynamics / Michael C. Kelley, with contributions from Rodney A. Heelis , xii, 487 p. : pp., Academic Press, 
San Diego :, 1989.

Kivelson, M. G., and C. T. Russell, Introduction to Space Physics, 1995.

Kletzing, C. A., and S. Hu, Alfvén wave generated electron time dispersion, Geophys. Res. Lett., 28, 693-696, doi:10.1029/2000GL012179, 2001.

Kletzing, C. A., G. Berg, M. C. Kelley, F. Primdahl, and R. B. Torbert, The electrical and precipitation characteristics of morning sector Sun-aligned auroral arcs, J. Geophys. Res., 101, 17,175-17,190, doi:10.1029/96JA00294, 1996.

Mallinckrodt, A. J., A numerical simulation of auroral ionospheric electrodynamics, J. Geophys. Res., 90, 409-417, doi:10.1029/JA090iA01p00409, 1985.

Mallinckrodt, A. J., and C. W. Carlson, On the anticorrelation of the electric field and peak electron energy within an auroral arc, J. Geophys. Res., 90, 399-408, doi: 10.1029/JA090iA01p00399, 1985.

Marklund, G., Auroral arc classification scheme based on the observed arc-associated electric field pattern, Planet. Space Sci., 32, 193-211, doi:10.1016/0032-0633(84)901545,1984 .

Marklund, G., I. Sandahl, and H. Opgenoorth, A study of the dynamics of a discrete auroral arc, Planet. Space Sci., 30, 179-197, doi:10.1016/0032-0633(82)90088-5, 1982.

Marklund, G., L. Blomberg, C. Fälthammar, and P. Lindqvist, On intense diverging electric fields associated with black aurora, Geophys. Res. Lett., 21, 1859-1862, doi: 10.1029/94GL00194, 1994.

Marklund, G., T. Karlsson, and J. Clemmons, On low-altitude particle acceleration and intense electric fields and their relationship to black aurora, J. Geophys. Res., 102, 17,509-17,522, doi:10.1029/97JA00334, 1997. 
Pfaff, R. F., In-situ measurement techniques for ionospheric research, pp. 459-551, European Geophysical Society, 1996.

Rees, M. H., Auroral ionization and excitation by incident energetic electrons, Planet. Space Sci., 11, 1209-+, doi:10.1016/0032-0633(63)90252-6, 1963.

Rees, M. H., On the interaction of auroral protons with the earth's atmosphere, Planet. Space Sci., 30, 463-472, doi:10.1016/0032-0633(82)90056-3, 1982.

Rees, M. H., Physics and Chemistry of the Upper Atmosphere, 1989.

Richmond, A. D., and J. P. Thayer, Ionospheric Electrodynamics: A Tutorial, pp. 131-+, The American Geophysical Union, 2000.

Sangalli, L., D. J. Knudsen, M. F. Larsen, T. Zhan, R. F. Pfaff, and D. Rowland, Rocketbased measurements of ion velocity, neutral wind, and electric field in the collisional transition region of the auroral ionosphere, Journal of Geophysical Research (Space Physics), 114, A04,306, doi:10.1029/2008JA013757, 2009.

Schott, L., Plasma Diagnostics. W. Lochte-Holtgreven, Ed. North-Holland, Amsterdam; Interscience (Wiley), New York, 1968, 668 pp., 1968.

Swift, D. W., Effective Height-Integrated Conductivity of the Ionosphere, J. Geophys. Res., 77, 1279-1285, doi:10.1029/JA077i007p01279, 1972.

Vasyliūnas, V. M., and P. Song, Meaning of ionospheric Joule heating, J. Geophys. Res., 110, A02,301, doi:10.1029/2004JA010615, 2005. 


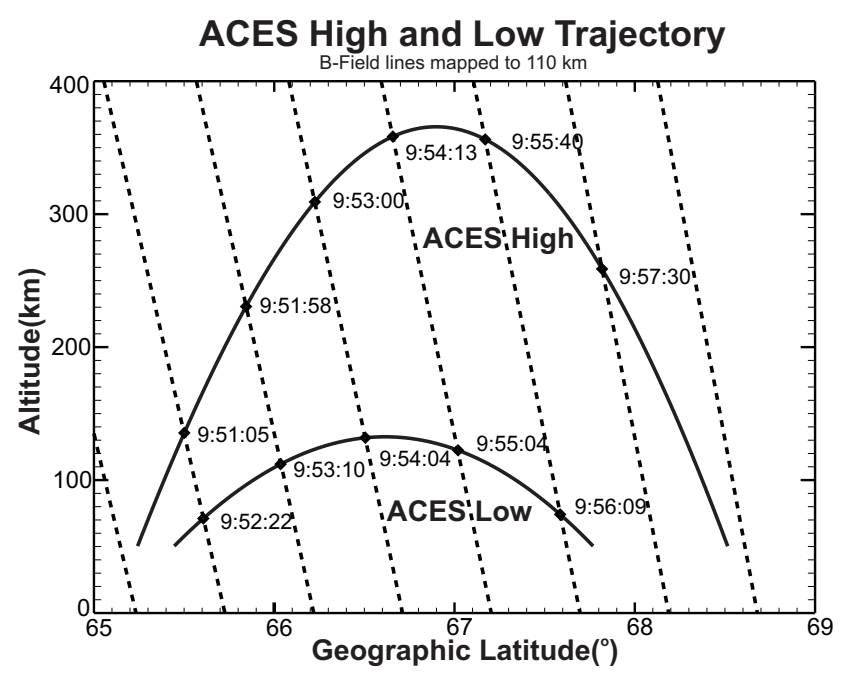

Figure 1. Altitude and geographic latitude were plotted, along with the locations of the magnetic field footpoints mapped to $110 \mathrm{~km}$. Times also indicate when both payloads respectively crossed the same magnetic field line. 

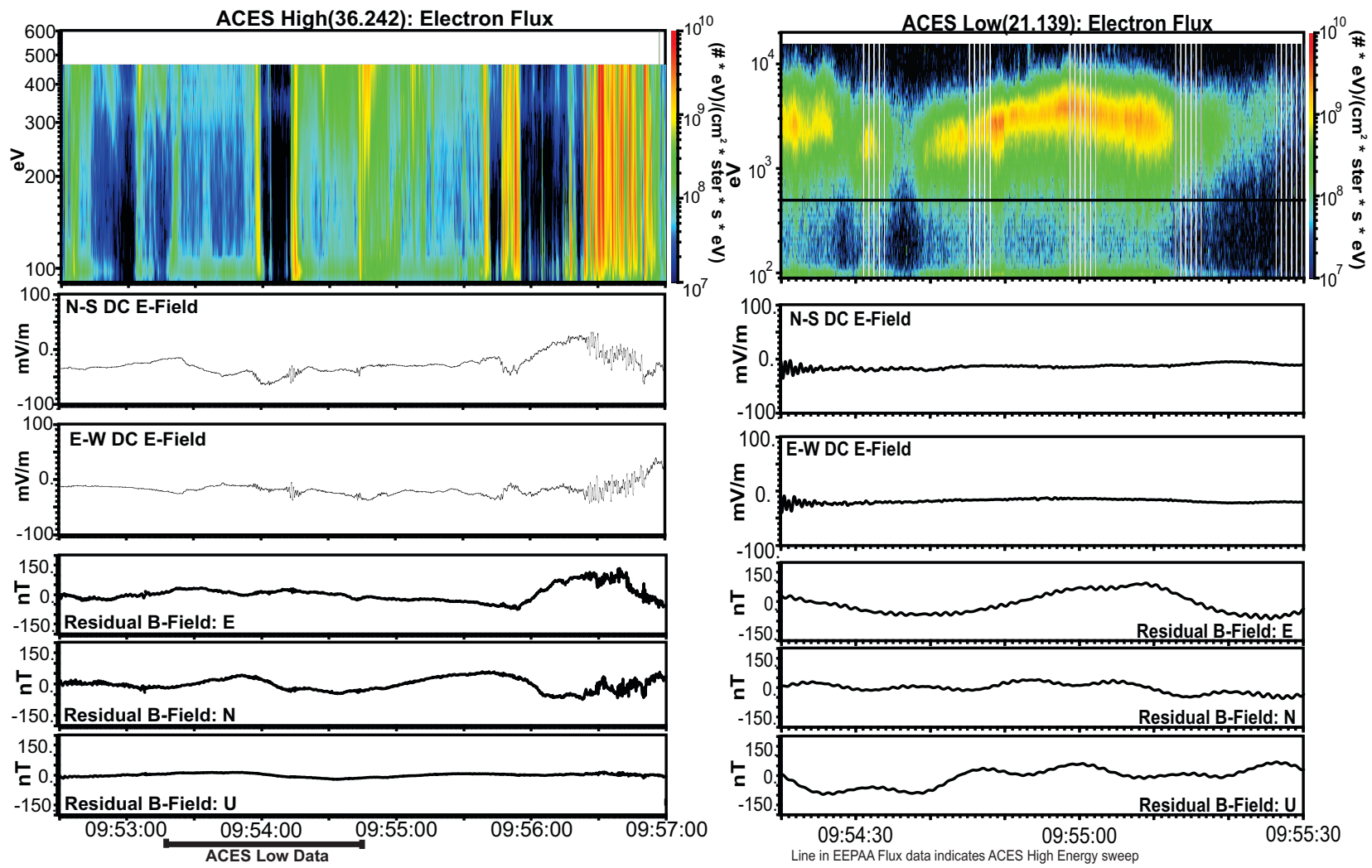

Figure 2. Results from both the ACES High and ACES Low payloads. Left Column:

ACES High Data; Right Column: ACES Low Data. Row 1: Differential Electron Energy Flux; Row 2-3: DC Electric Field Data; Row 4-6: Residual Magnetic Field Components 


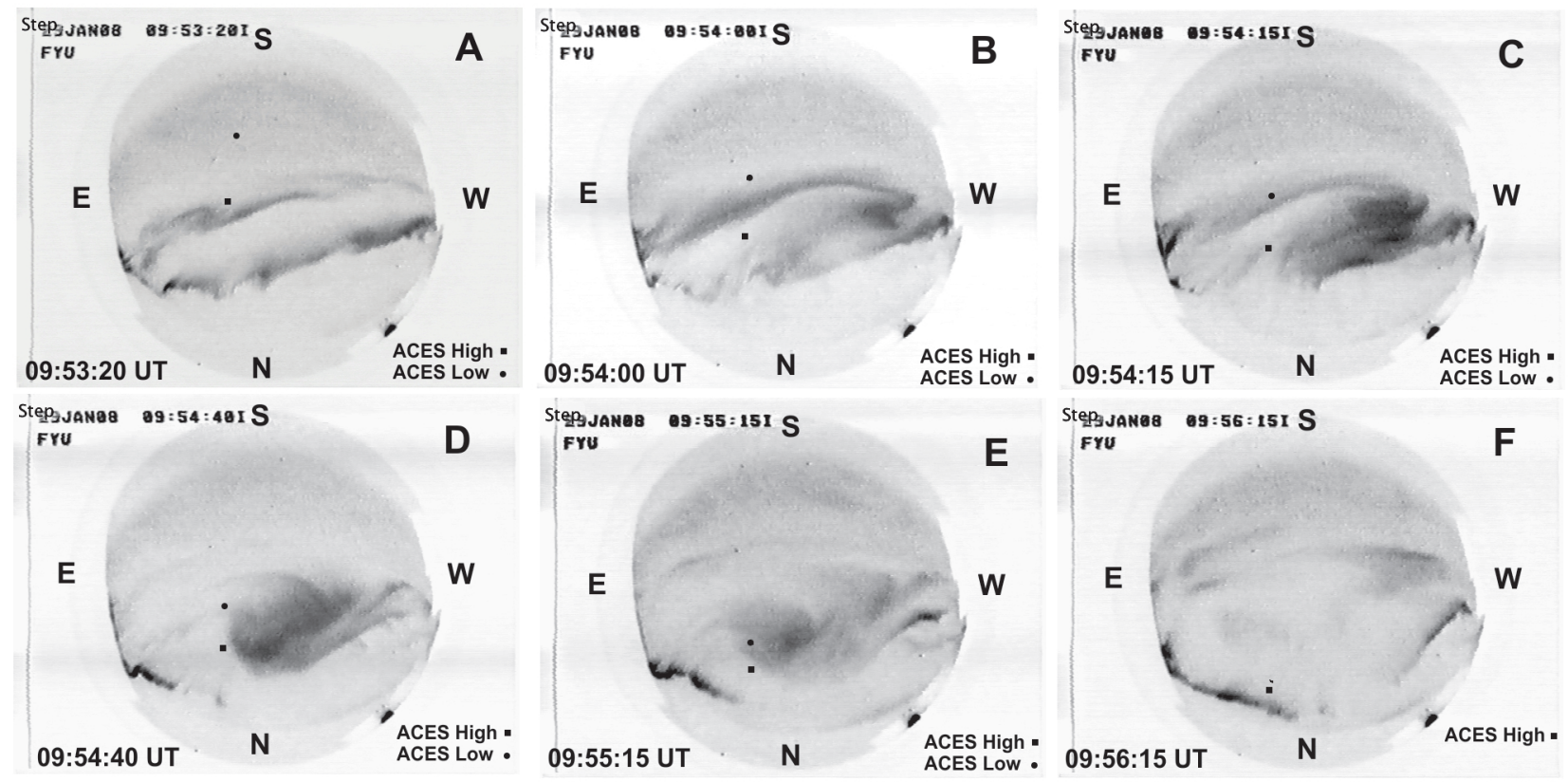

Figure 3. The images from the Fort Yukon all-sky imager detailing the evolution of the auroral event on 29 January 2009. Labels are presented representing the cardinal directions. ACES High and Low are represented by a square and dot, respectively 

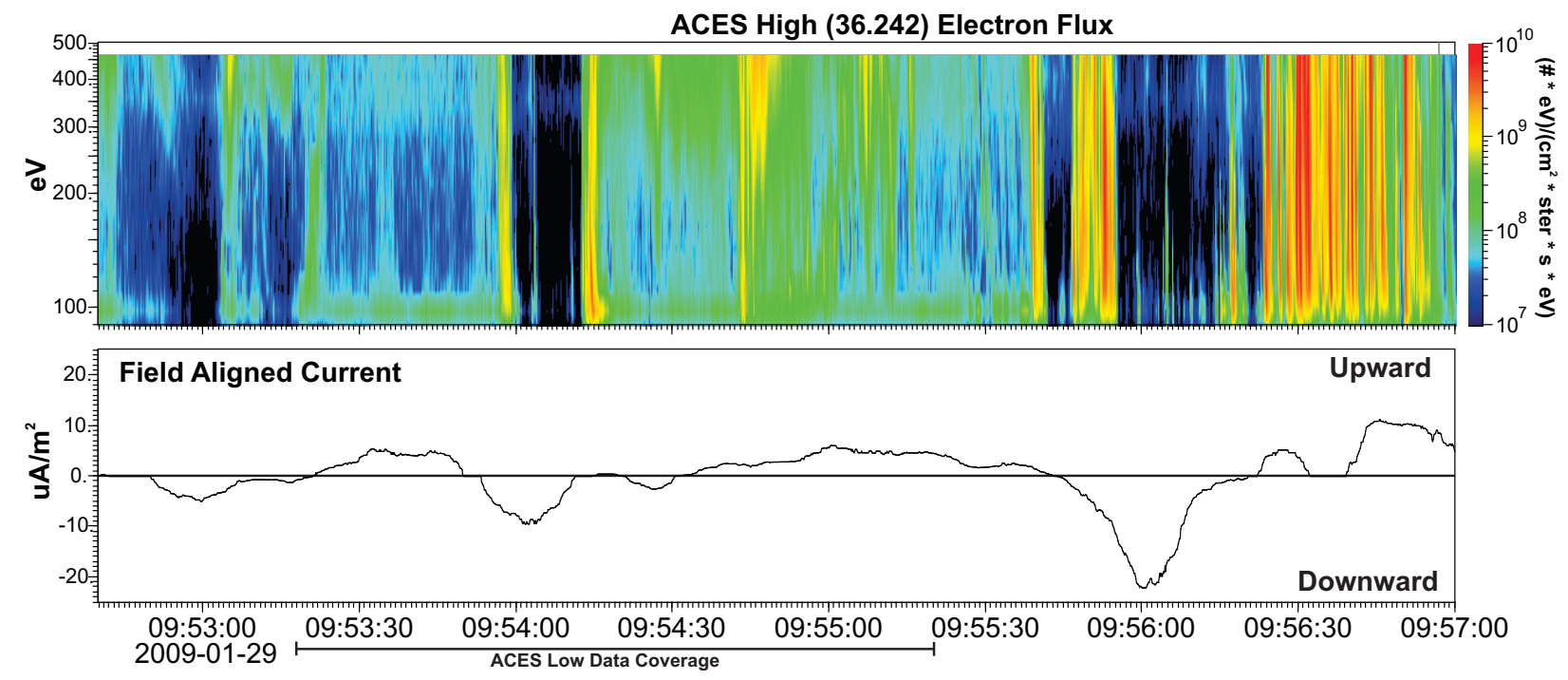

Figure 4. Results from the calculation of field-aligned current detailing the correlations between the differential electron energy flux and the upward and downward directed fieldaligned current data.

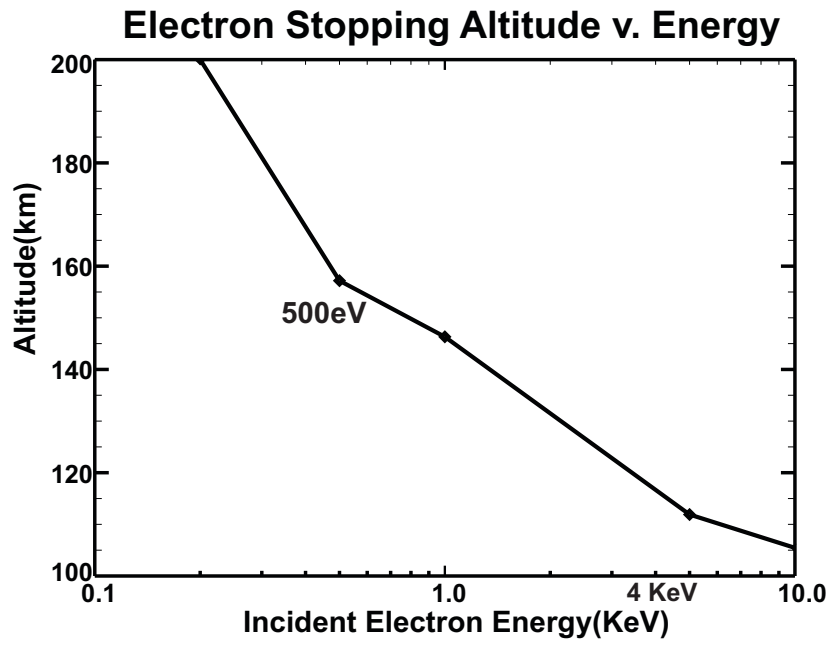

Figure 5. The results from the stopping altitude calculation which show that precipitating electrons at $500 \mathrm{eV}$ become indistinguishable at about $160 \mathrm{~km}$ from background ionospheric electrons. 